\title{
Video-Assisted Thoracic Surgery Intrathoracic Anastomosis Technique
}

\author{
Yong Won Seong, M.D. \\ Department of Thoracic and Cardiovascular Surgery, Seoul Metropolitan Government-Seoul National University Boramae Medical Center, Seoul National \\ University College of Medicine, Seoul, Korea
}

\section{ARTICLE INFO}

Received July 20, 2021

Accepted July 23, 2021

Corresponding author

Yong Won Seong

Tel $82-2-870-2294$

Fax 82-2-831-2826

E-mail ARQJOKER@gmail.com

ORCID

https://orcid.org/0000-0002-3218-468X
The extracorporeal anastomosis technique for video-assisted thoracoscopic surgery (VATS) intrathoracic esophagogastric anastomosis is a convenient, easy technique to use in VATS esophagectomy. The surgeon can assess the viability and the status of the gastric conduit, and the introduction of a circular stapler can be easily done under direct vision extracorporeally, enabling easy and simple VATS intrathoracic anastomosis between the esophagus and the gastric conduit.

Keywords: Esophageal neoplasms, Minimally invasive surgery, Anastomosis, Video-assisted thoracic surgery

\section{Introduction}

Since Cuschieri et al. [1] reported the first series of 5 cases of minimally invasive esophagectomy (MIE), MIE has become an established and widely accepted approach for treating esophageal malignancies over the past 2 decades. It has been reported that when MIE is performed at high-volume centers, it shows similar clinical outcomes in terms of morbidity, mortality, and other oncologic outcomes to those of open esophagectomy, with clear advantages due to the minimally invasive approach [2]. Although MIE is technically demanding and involves a definite learning curve for surgeons, it is nonetheless an excellent approach for esophagectomy. The advantages of the minimally invasive approach include less postoperative pain and blood loss, a reduced incidence of pulmonary complications, and shorter hospital stays [3-5]. MIE techniques have been developed and evolved significantly from the initially used hybrid approaches (thoracoscopy combined with laparotomy or laparoscopy combined with thoracoscopy) to the totally minimally invasive approaches (thoracoscopy combined with laparoscopy, totally robotic) in current use. The esophagogastric anastomosis can be placed at cervical or intrathoracic location. The cervical anastomosis has been reported to pose a higher risk of anastomotic leakage, stricture, recurrent laryngeal nerve injury, and swallowing dysfunction, but with less severe morbidity from any leakage that does occur [6-8]. In contrast, transthoracic anastomosis has been reported to have more morbid consequences when an anastomotic leakage occurs [9]. When the 3-field MIE McKeown operation is performed, every surgeon can assess the viability of the gastric conduit under direct vision. However, when the MIE Ivor Lewis operation is performed, direct visual assessment of the gastric conduit is very difficult, as it relies only on the quality of the thoracoscopic vision. Therefore, some reports have described successfully assessing gastric conduit viability by using fluorescence dyes such as indocyanine green [10-12]. However, these novel techniques of assessing gastric conduit viability by using novel imaging systems are not always available at relatively small-volume centers, and this discrepancy may explain why better clinical outcomes have been reported from large-volume centers. We would like to introduce a hybrid Ivor Lewis MIE technique (thoracoscopy combined with laparotomy) for video-assisted thoracoscopic surgery (VATS) extracorporeal intrathoracic esophagogastric anastomosis that can be easily performed and enables surgeons to assess the viability of the gastric conduit under direct vision. 


\section{Preoperative workup and planning}

The preoperative workup is not different from that of patients undergoing open esophagectomy. Esophagogastroduodenoscopy is always performed to confirm the precise location of the tumor, its proximal and distal margins, the presence of Barrett esophagus, and the status of the stomach for the conduit. Computed tomography of the chest and abdomen, endoscopic ultrasonography, positron emission tomography-computed tomography, pulmonary function testing, and a cardiac function evaluation are performed. Patients with advanced clinical stages who have undergone neoadjuvant treatments (chemotherapy or chemoradiation) are also similarly prepared. Patients with upper esophageal cancers undergo the McKeown operation and are not candidates for this technique. Smoking cessation for 2 weeks or more is mandatory for every patient to minimize postoperative pulmonary complications. Antiplatelet agents such as aspirin or clopidogrel are stopped 5 days before surgery.

\section{Operative techniques}

\section{Anesthesia}

All anesthetic procedures are performed or supervised by anesthesiologists who have experienced 100 or more esophagectomy cases. For all patients, endotracheal intubation for single-lung ventilation is performed with a left-sided double-lumen endotracheal tube (Shiley endotracheal tube; Medtronic, Minneapolis, MN, USA). The diameter of the double-lumen tube is determined according to the inner diameter of the left main bronchus of the patient on preoperative computed tomography. Patients are monitored with pulse oximetry, electrocardiography, and invasive and non-invasive measurements of arterial blood pressure. Central venous catheter insertion is not mandatory, and is mostly performed only in patients with advanced tumor stages or patients who have undergone neoadjuvant chemoradiation. A nasogastric Levin tube is always placed before surgery.

\section{Abdominal phase: gastric mobilization}

The patient is placed in the supine position. The arms can be abducted or not. The surgeon operates on the right side of the patient.

The abdomen is mapped, and a midline laparotomy incision is made from below the xiphoid process to the umbili- cus. The abdomen is thoroughly staged by inspecting and palpating the liver, peritoneum, and omentum to rule out any metastatic disease. A large wound protector with an OmniFlex retractor (Gadelius Medical K.K., Tokyo, Japan) affords an excellent surgical field (Fig. 1).

After identifying the gastrocolic omentum, the lesser sac is entered by creating a window at the greater omentum. Division of the omental branches of the gastroepiploic artery is performed. The right gastroepiploic arcade can be identified clearly by inspection and palpation, and must be protected securely during this procedure.

Dissection is continued along the greater curve of the stomach until the gastroepiploic arcade ends, and then division of the short gastric vessels is performed using energy devices: ultrasonic shears (Harmonic HD 1000i; Ethicon, Raritan, NJ, USA) or bipolar devices (LigaSure; Medtronic). Especially when dividing the short gastric vessels by these energy devices, making the division closer right next to the gastric conduit is helpful for minimizing unsuspected bleeding after division with an energy device. Retrogastric attachments are divided after completing the division of the short gastric vessels.

Dissection of the pyloroantral region must be carefully and meticulously done because any injury to the proximal right gastroepiploic artery can result in a very frustrating situation. A partial or complete Kocher maneuver is sometimes needed for adequate mobilization.

The gastrohepatic ligament is opened. Triangular resection of the gastrohepatic ligament is helpful, affording ade-

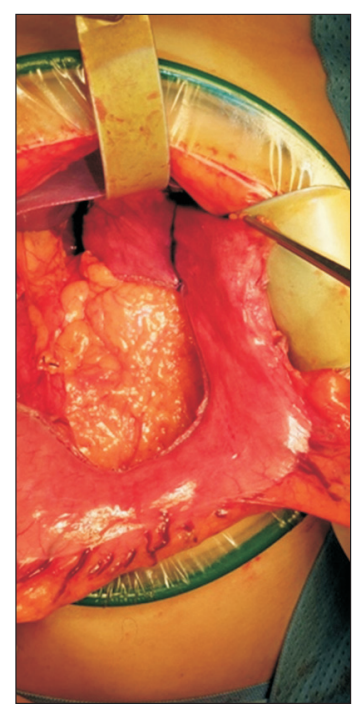

Fig. 1. Surgical view of midline laparotomy and gastric tube formation. Note that the $4-\mathrm{cm}$ length of the uppermost fundus is left intact, not fully divided. 
quate access for sweeping the lymph nodes (LNs) and fatty tissue, including the celiac LNs, left gastric LNs, and LNs along the splenic artery and the superior border of the pancreas. The left gastric artery and vein can be easily identified and dissected after this LN dissection. The left gastric artery and vein are divided after proximal double-ligation or double clipping using Weck Hem-o-Lok clips (Teleflex, Morrisville, NC, USA).

Dissection of the crus and mobilization of the distal abdominal esophagus are performed. The distal esophagus is retracted anteriorly, posteriorly, and bilaterally, and it then can be easily mobilized. Division of the crus is helpful for subsequent passage of the gastric conduit. A width of 3 fingerbreadths is adequate for most of the gastric conduit passage. At this point, the distal esophagus, gastric fundus, and antrum should be completely freely mobilized.

\section{Abdominal phase: gastric tube formation}

The Levin tube is requested to be withdrawn until $20 \mathrm{~cm}$ at the nasal orifice. The gastric tubing is performed before the pyloric drainage procedure (pyloromyotomy/pyloroplasty) and feeding jejunostomy, providing time to assess the viability of the gastric conduit.

The stomach is well stretched by the hands of the surgeon and the first assistant, and then the stomach is first divided across the antrum with an endoscopic purple stapler (Endo-GIA 60 mm Reload with Tri-staple Technology; Medtronic) or an endoscopic green stapler (Ethicon+Stapler with GST Reloads; Ethicon). When using the Ethicon stapler, we always hold the jaws in place for 15 seconds prior to firing for better compression and staple formation.

The subsequent stapling continues toward the fundus, maintaining a conduit width of $4-5 \mathrm{~cm}$, parallel to the greater curvature. The stapling finishes with $4 \mathrm{~cm}$ of the fundus left intact, which later becomes an extracorporeal passage route for an EEA Circular Stapler (Medtronic). This site eventually needs to be stapled intrathoracically in the thoracic phase, so this area is marked with a surgical marker pen anteriorly and posteriorly (Fig. 1).

Reinforcing seromuscular sutures can be made along the stapler line, according to the surgeon's preference. We prefer to make seromuscular sutures at least at every junction of the stapler lines.

\section{Abdominal phase: pyloric drainage procedures and feeding jejunostomy}

Any pyloric drainage procedure-pyloromyotomy, pylo- roplasty, or botulinum toxin injection-can be performed following the surgeon's preference. We usually perform a pyloromyotomy with an electrocautery amplitude of 5-10. The innermost layer of the pyloric muscle is divided by carefully removing the muscle fibers with a mosquito clamp, rather than by dividing the muscle through electrocautery.

Feeding jejunostomy tube insertion can be also performed according to the surgeon's preference. We usually perform feeding jejunostomy tube insertion in a Witzel fashion.

The laparotomy wound closure is performed layer-bylayer. We usually do not place any intraabdominal drains such as a Jackson-Pratt drain.

\section{Thoracoscopic phase (VATS phase): positioning and port placement}

The patient is repositioned into the left lateral decubitus position for the thoracoscopic phase. The location of the double-lumen tube is reconfirmed by the anesthesiologist. The operating surgeon stands on the right side of the patient, while the assistant stands on the left side of the table.

Four thoracoscopic ports are used. The right scapula and the locations for the ports are shown in Fig. 2A. A $5-\mathrm{cm}$ utility incision is made on the fifth rib on the anterior axillary line, and then a $4-\mathrm{cm}$ segment of the fifth rib is resected using a periosteal elevator and a rib cutter (Fig. 2B). Sharp resection margins of the ribs are made blunt by using a rongeur. A $14-\mathrm{mm}$ incision is made on the seventh intercostal space, along the midaxillary line, for a $12-\mathrm{mm}$
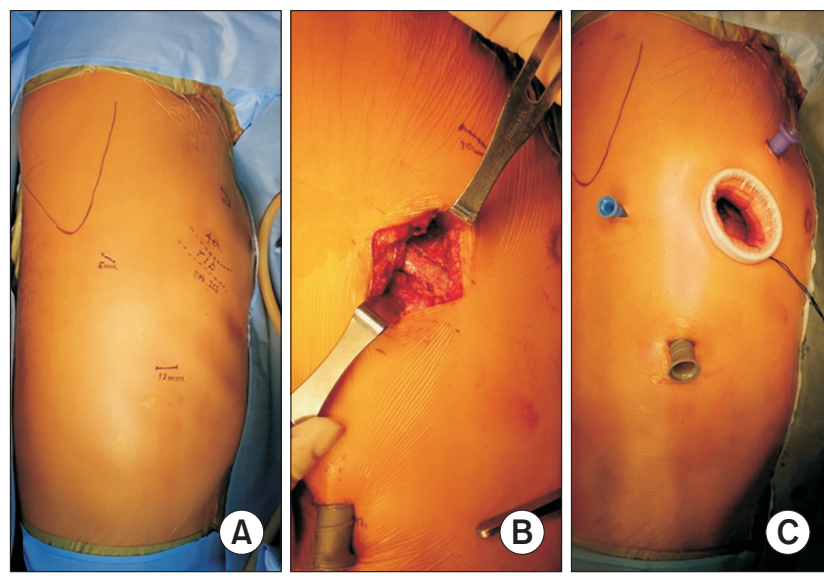

Fig. 2. (A) The location of the ports to be placed is shown by a marker pen. (B) A 4-cm segment of the fifth rib is resected for subsequent easy passage of the extracorporeal procedures. (C) Image after the port placement. 
trocar. A 7-mm incision is made on the sixth intercostal space, anterior to the scapular tip, for a 5 -mm trocar. Finally, a $12-\mathrm{mm}$ incision is made on the third intercostal space, along the anterior axillary line, for a $10-\mathrm{mm}$ trocar, which is used as an assistant port. The completed port placement is shown in Fig. 2C.

\section{Thoracoscopic phase (VATS phase): esophagectomy and LN dissection}

The mediastinal pleura is vertically opened along the course of the esophagus. The azygous vein is divided using a vascular endoscopic stapler. The posterior stump of the divided azygous vein is sutured to the posterior mediastinal pleura with a 3-0 silk suture, and the suture is then tacked and clipped with a 5-mm Weck Hem-o-Lok. This affords a better surgical view of the esophagus. This tacking suture is later removed before wound closure.

Esophagectomy using an energy device is performed. Whether or not to remove the entire mesoesophagus, including the thoracic duct, relies on the surgeon's preference. We usually keep the thoracic duct intact. After finishing esophagectomy, complete total 2-field $\mathrm{LN}$ dissection is performed. The bilateral paraesophageal, bilateral recurrent laryngeal, subcarinal, hilar, and aortopulmonary window LNs are completely removed.

\section{Thoracoscopic phase (VATS phase): extracorporeal introduction of the circular stapler}

A purse-string suture is made at the upper thoracic esophagus over the azygous vein level with a 2-0 polyester suture (Ethibond EXCEL; Ethicon). Then, a vertical esophagotomy incision is made $5-10 \mathrm{~mm}$ below the pursestring suture. The anvil for a $28-\mathrm{mm}$ or $25-\mathrm{mm}$ circular stapler (EEA Circular Stapler; Medtronic) is vertically inserted into the esophagotomy. The anvil is then introduced into the upper thoracic esophagus through the purse-string suture. When introducing the anvil upward, keeping the anvil vertically while pushing the anvil upward enables us to pass the anvil through the purse-string suture more easily (Fig. 3). After the anvil passes the purse-string suture, the anvil is rotated, and then the suture is tied down.

The proximal esophagus is divided right below the previously tied-down purse-string suture, then is brought out extracorporeally through the utility incision and tied down with a 2-0 silk ligature.

The gastric conduit is pulled up into the thoracic cavity under thoracoscopic vision, and is then brought out extracorporeally through the utility incision. At this stage, the surgeon can assess the viability of the gastric conduit under direct vision. The surgeon can identify the line that was previously marked during the abdominal phase. This line later will become the final stapler line after finishing the EEA stapling. The EEA stapler puncture site is shown by a yellow arrow in Fig. $4 \mathrm{~A}$. We keep at least a $4-\mathrm{cm}$ distance between this puncture site and the final stapling line in order to maximize the submucosal blood flow around

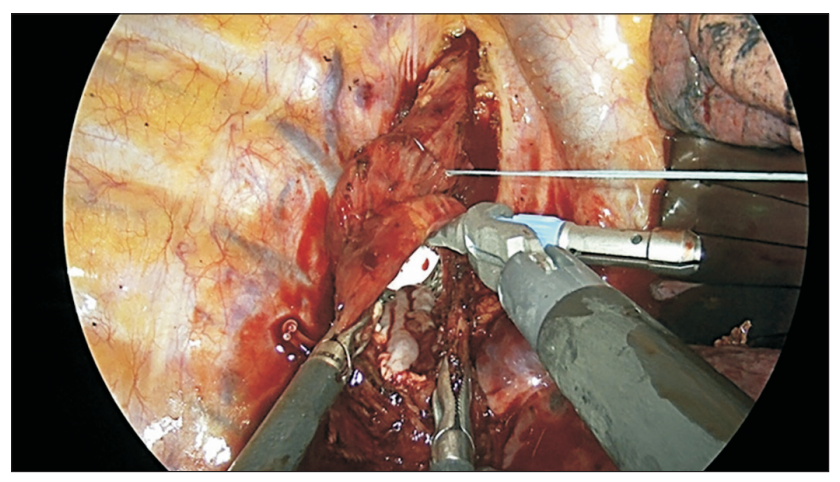

Fig. 3. Intrathoracic anvil insertion is shown. Gentle vertical upward introduction of the anvil, not rotating below the purse-string suture, is important for easier passage of the anvil through the purse-string suture.
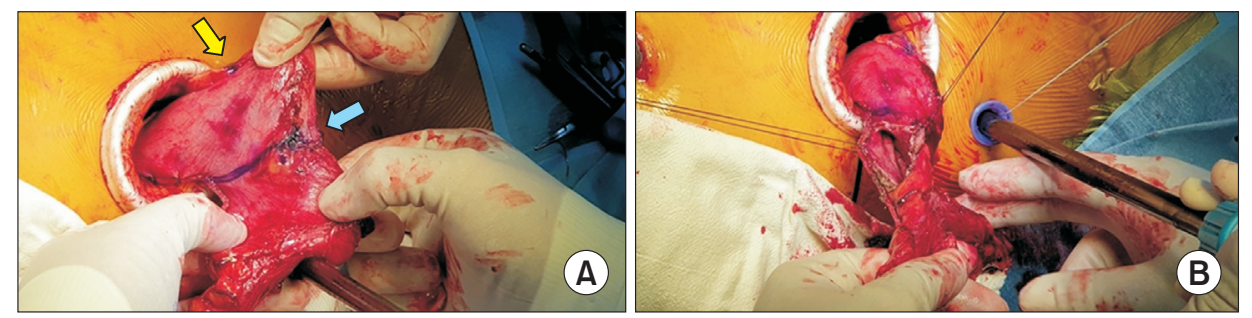

Fig. 4. The gastric conduit is extracted through the utility incision, followed by (A) extracorporeal assessment of the conduit and (B) gastrotomy with tacking sutures for introducing the EEA stapler (Medtronic, Minneapolis, MN, USA). Note the 4-cm distance between the yellow and blue arrows in Fig. 4A. Keeping this distance between the circular stapler line and the linear stapler line is important for preventing anastomosis site ischemia and leakage. 
the circular stapling site to prevent anastomotic leakage (Fig. 4A). A 4-cm gastrotomy incision is made right next to the stapler line at the remnant fundus. The gastric and esophageal blood and contents are removed by a sucker, and each opened side of the fundus is then tacked with a 2-0 silk suture (Fig. 4B).

An EEA stapler is introduced through the gastrotomy, and the fundus is then punctured (Fig. 5A). The punctured gastric conduit and the EEA can be easily and smoothly reintroduced into the thoracic cavity through the utility incision. The previous segmental resection of the fifth rib affords adequate space for these extracorporeal procedures. It is important to gently pull the previously placed 2 silk tacking sutures to maintain the EEA at the proper position inside the conduit (Fig. 5B).

\section{Thoracoscopic phase (VATS phase): intrathoracic anastomosis}

With the reintroduction of the conduit intrathoracically, the VATS camera affords an excellent view of the previously placed anvil and the introduction of the EEA and the conduit (Fig. 6A). Once the sharp end of the EEA tip enters the anvil, the cervical vertebra and the thoracic inlet serve as a supporting wall; therefore, docking between the main EEA body and the anvil can be easily performed (Fig. 6B). After stapling is finished, the EEA and the anvil are gently retrieved and the donut-shaped margins of the cross-section at the removed anvil are inspected for completeness and sent to the pathologist for a frozen section.

The surgeon can check the completeness of the anasto- mosis by entering the thoracoscope through the gastrotomy site where the EEA entered, but this is not routinely performed. The previously marked linear line on the conduit is stapled with the same endoscopic stapler loads that were used for conduit formation.

The nasogastric Levin tube is now reinserted and fixed at $45 \mathrm{~cm}$ at the nasal orifice. This usually results in an adequate location of the tip of the nasogastric tube, just above the pylorus. Multiple interrupted seromuscular sutures (3-0 silk sutures) are made over the final linear stapled line.

Repair of the upper mediastinal pleura is always performed using multiple interrupted 3-0 silk sutures (Fig. 7). We consider this procedure very important, since this closure of the pleura-especially around the anastomosis site-provides protection from viable tissue, and even if there is a leakage postoperatively it can be successfully treated by endoluminal vacuum-assisted closure therapy, with the closed mediastinal pleura serving as a viable flap layer. The previously tacked posterior azygous vein stump is released.

The gastric conduit below the azygous vein is adequately adjusted; if there is any redundancy, the redundant portion is put back into the abdominal cavity. When an adequate position of the conduit is confirmed, the conduit is fixed to the posterior mediastinal pleura with 3 or 4 interrupted 3-0 silk sutures.

Irrigation of the thoracic cavity with warm distilled water is done. A single 28F straight soft straight chest tube is inserted, and wound closure is performed layer-by-layer. The patient is extubated and transferred to the intensive care unit.
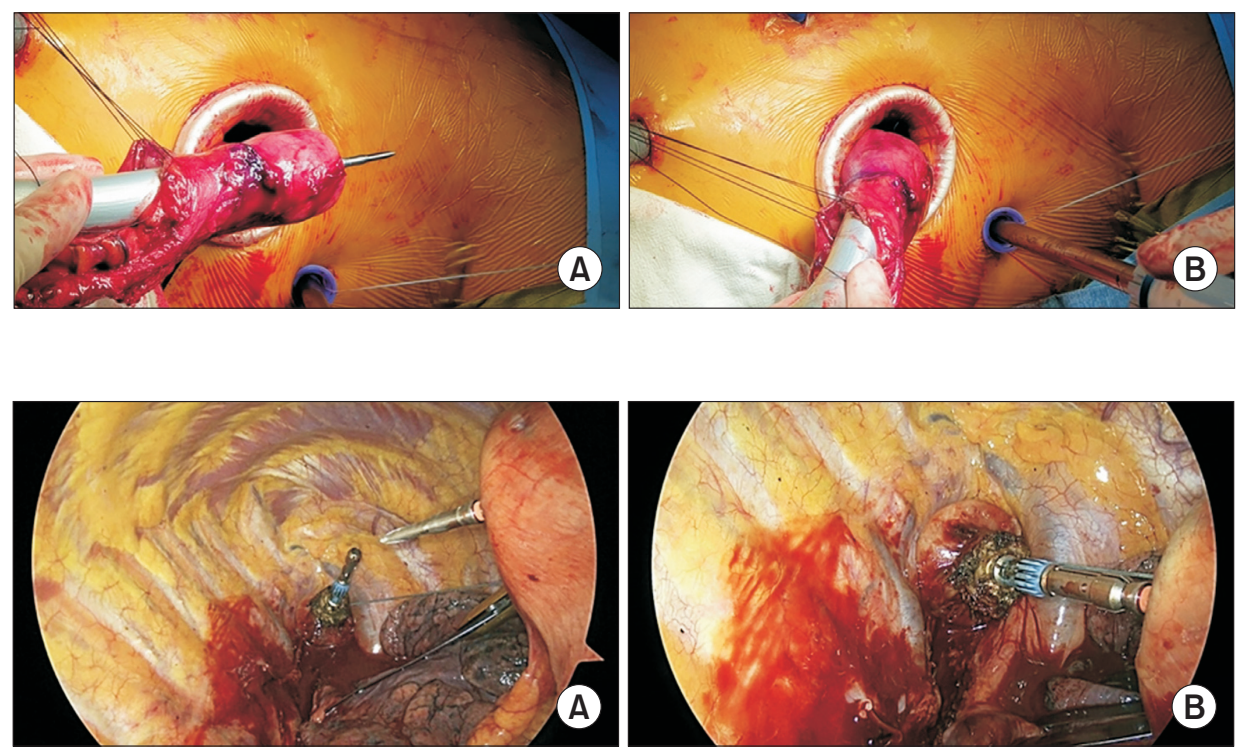

Fig. 6. Video-assisted thoracoscopic surgery intrathoracic anastomosis is performed. With the cervical vertebra and the small thoracic inlet serving as a supporting table, docking between the anvil and the body of the EEA stapler (Medtronic, Minneapolis, MN, USA) can be easily done. 


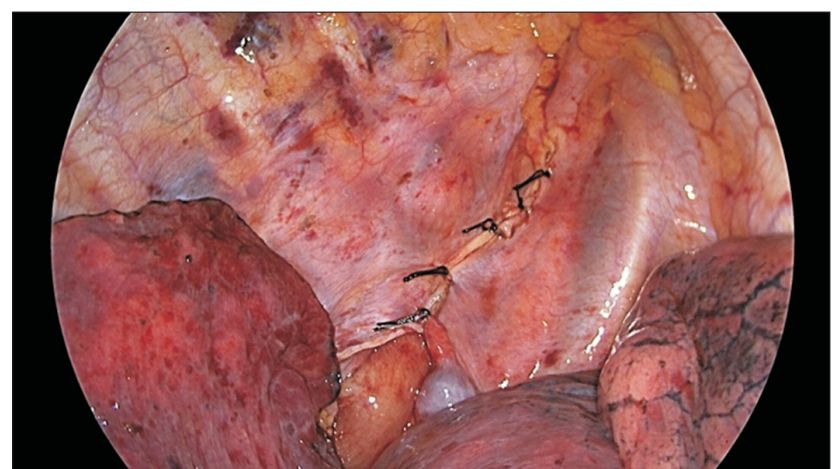

Fig. 7. The upper portion of the mediastinal pleura over the azygous vein level is suture-repaired with multiple interrupted 3-0 silk sutures. This closure of the upper pleura is an easy procedure that takes only a couple of minutes, and serves as a viable tissue barrier around the anastomosis site.

\section{Discussion}

\section{Lateral decubitus versus prone position}

Luketich et al. [13] reported a large series of total MIE (thoracoscopy combined with laparoscopy) in 222 patients with impressive clinical outcomes, including low mortality and pulmonary complication rates of $1.4 \%$ and $7.7 \%$, respectively. In this series, all the cases were performed with the patients in the lateral decubitus position, as was done for most MIE procedures in this period [13]. However, there were concerns regarding pulmonary complications, as the lateral decubitus requires complete lung collapse and therefore often causes serious pulmonary complications. Palanivelu et al. [14] reported a large series of thoracoscopic mobilization of the esophagus in the prone position with excellent clinical outcomes, as shown by mortality and pulmonary complication rates of $1.54 \%$ and $1.54 \%$, respectively. In their systematic review and pooled analysis, Markar et al. [15] reported that prone MIE is superior to the lateral decubitus position, with reduced pulmonary complications, lower estimated blood loss, and increased LN harvest. However, urgent conversion to lateral thoracotomy is difficult when the patient is in the prone position. Therefore, a modified semiprone position was reported and adopted by many surgeons [16]. In our technique, we perform the procedure with the patient in the lateral decubitus position, but we never grab or clamp the lung for traction. We only add gentle pushing to the lung with an endoscopic fan retractor or an endoscopic peanut.

\section{Hand-sewn versus side-to-side linear stapling versus end-to-end circular stapling}

In the days of open esophagectomy, the hand-sewn anastomosis was preferred by many surgeons, with good results [17]. There have been many reports describing favorable clinical outcomes of hand-sewn MIE performed using a robot, with the aid of the articulating robotic arms and the 3-dimensional imaging system [18]. However, there have not been many reports of minimally invasive hand-sewn anastomosis due to its technical difficulty with instruments without articulation $[19,20]$.

The linear stapling technique is a side-to-side anastomosis between the esophagus and the conduit, which has been reported to be functionally favorable with a larger diameter of the anastomosis that offers easy passage of the intraluminal content and reduced circular pressure at the anastomosis site. These factors might explain the low incidence of anastomotic leakage and strictures [21-23].

Since Fain et al. [24] reported the first use of a circular stapling apparatus in colorectal cancer surgery in 1975, the device and the technique have evolved. Since this technique is the longest-known and best-developed, it is wellstandardized and relatively easy to learn. However, there are still many technically important points, and the learning curve is steep for novice surgeons. For example, inserting an EEA anvil thoracoscopically is very difficult if the proximal esophagus is completely divided. Vertical esophagotomy below the purse-string suture and an upward vertical entrance of the EEA anvil is a technique that almost always makes the insertion of a $28-\mathrm{mm}$ anvil possible. It is also very important to minimize the size of the tied esophageal stump of the anvil before docking of the EEA. If the tied esophageal stump is large, it can be squeezed out laterally between the stapler lines when docking, which can result in an area of incomplete stapling.

\section{Extracorporeal anastomosis versus intracorporeal anastomosis}

Extracorporeal anastomosis is a well-known and established concept in the area of general surgery. For example, right-side hemicolectomy for right colonic cancer is commonly performed [25], and laparoscopic-assisted right hemicolectomy with an extracorporeal anastomosis is the standard technique in most countries. The current standard procedure for laparoscopic extracorporeal anastomosis requires adequate positioning of the utility incision, mobilization of the colon, and mesenteric traction in order 
to extract the ileum and ascending colon; this can result in surgical trauma, leading to postoperative morbidities such as prolonged ileus, pain associated with pulmonary function, and wound infection with a prolonged length of stay [25-27]. Furthermore, the extracorporeal anastomosis technique for laparoscopic-assisted right hemicolectomy requires the location of the extraction wound to be in the upper/mid-abdomen, which is known to result in increased postoperative pain when compared with the wound located in the lower abdomen [27]. Van Oostendorp et al. [28] reported in their meta-analysis that intracorporeal anastomosis was associated with less morbidity and a shorter length of stay, suggesting that it enabled a faster recovery than occurred after extracorporeal anastomosis.

Although extracorporeal anastomosis is reported to have limitations compared with intracorporeal anastomosis in right hemicolectomy, the extracorporeal anastomosis technique in VATS intrathoracic anastomosis after esophagectomy is different. The location of the utility incision is not different from that of the conventional VATS intracorporeal anastomosis technique. No additional mobilization of the gastric conduit or Kocher maneuver is required to extract the conduit. The surgeon can thoroughly assess the viability of the conduit under direct vision and palpation, and the introduction of the EEA and final conduit design can also be accurately planned extracorporeally. Kim et al. [29] reported 66 patients with esophageal cancer who underwent VATS Ivor Lewis operations using the extracorporeal anastomosis technique. They presented excellent clinical outcomes without any anastomotic leakage or stricture.

\section{Conclusion}

Extracorporeal anastomosis for VATS intrathoracic esophagogastric anastomosis is a convenient, easy technique without any requirement for a robotic system, articulating instruments, or any other novel devices or instruments; therefore, this technique can be learned and adopted by surgeons who are newly starting to perform MIE.

\section{Conflict of interest}

No potential conflict of interest relevant to this article was reported.

\section{ORCID}

Yong Won Seong: https://orcid.org/0000-0002-3218-468X

\section{References}

1. Cuschieri A, Shimi S, Banting S. Endoscopic oesophagectomy through a right thoracoscopic approach. J R Coll Surg Edinb 1992; 37:7-11.

2. Levy RM, Trivedi D, Luketich JD. Minimally invasive esophagectomy. Surg Clin North Am 2012;92:1265-85.

3. Xiong WL, Li R, Lei HK, Jiang ZY. Comparison of outcomes between minimally invasive oesophagectomy and open oesophagectomy for oesophageal cancer. ANZ J Surg 2017;87:165-70.

4. Nagpal K, Ahmed K, Vats A, et al. Is minimally invasive surgery beneficial in the management of esophageal cancer?: a meta-analysis. Surg Endosc 2010;24:1621-9.

5. Verhage RJ, Hazebroek EJ, Boone J, van Hillegersberg R. Minimally invasive surgery compared to open procedures in esophagectomy for cancer: a systematic review of the literature. Minerva Chir 2009;64: 135-46.

6. Martin RE, Letsos P, Taves DH, Inculet RI, Johnston H, Preiksaitis HG. Oropharyngeal dysphagia in esophageal cancer before and after transhiatal esophagectomy. Dysphagia 2001;16:23-31.

7. Hulscher JB, Tijssen JG, Obertop H, van Lanschot JJ. Transthoracic versus transhiatal resection for carcinoma of the esophagus: a meta-analysis. Ann Thorac Surg 2001;72:306-13.

8. Easterling CS, Bousamra M 2nd, Lang IM, et al. Pharyngeal dysphagia in postesophagectomy patients: correlation with deglutitive biomechanics. Ann Thorac Surg 2000;69:989-92.

9. Hulscher JB, van Sandick JW, de Boer AG, et al. Extended transthoracic resection compared with limited transhiatal resection for adenocarcinoma of the esophagus. N Engl J Med 2002;347:1662-9.

10. Luo RJ, Zhu ZY, He ZF, Xu Y, Wang YZ, Chen P. Efficacy of indocyanine green fluorescence angiography in preventing anastomotic leakage after McKeown minimally invasive esophagectomy. Front Oncol 2021;10:619822.

11. Ladak F, Dang JT, Switzer N, et al. Indocyanine green for the prevention of anastomotic leaks following esophagectomy: a meta-analysis. Surg Endosc 2019;33:384-94.

12. Schlottmann F, Patti MG. Evaluation of gastric conduit perfusion during esophagectomy with indocyanine green fluorescence imaging. J Laparoendosc Adv Surg Tech A 2017;27:1305-8.

13. Luketich JD, Alvelo-Rivera M, Buenaventura PO, et al. Minimally invasive esophagectomy: outcomes in 222 patients. Ann Surg 2003; 238:486-95.

14. Palanivelu C, Prakash A, Senthilkumar R, et al. Minimally invasive esophagectomy: thoracoscopic mobilization of the esophagus and mediastinal lymphadenectomy in prone position: experience of 130 patients. J Am Coll Surg 2006;203:7-16.

15. Markar SR, Wiggins T, Antonowicz S, Zacharakis E, Hanna GB. Minimally invasive esophagectomy: lateral decubitus vs. prone positioning; systematic review and pooled analysis. Surg Oncol 2015; 
24:212-9.

16. Seesing MF, Goense L, Ruurda JP, Luyer MD, Nieuwenhuijzen GA, van Hillegersberg R. Minimally invasive esophagectomy: a propensity score-matched analysis of semiprone versus prone position. Surg Endosc 2018;32:2758-65.

17. Cerfolio RJ, Bryant AS, Canon CL, Dhawan R, Eloubeidi MA. Is botulinum toxin injection of the pylorus during Ivor Lewis [corrected] esophagogastrectomy the optimal drainage strategy? J Thorac Cardiovasc Surg 2009;137:565-72.

18. Plat VD, Stam WT, Schoonmade LJ, Heineman DJ, van der Peet DL, Daams F. Implementation of robot-assisted Ivor Lewis procedure: robotic hand-sewn, linear or circular technique? Am J Surg 2020; 220:62-8.

19. Cadiere GB, Dapri G, Himpens J, Fodderie L, Rajan A. Ivor Lewis esophagectomy with manual esogastric anastomosis by thoracoscopy in prone position and laparoscopy. Surg Endosc 2010;24:1482-5.

20. Watson DI, Davies N, Jamieson GG. Totally endoscopic Ivor Lewis esophagectomy. Surg Endosc 1999;13:293-7.

21. Yanni F, Singh P, Tewari N, et al. Comparison of outcomes with semi-mechanical and circular stapled intrathoracic esophagogastric anastomosis following esophagectomy. World J Surg 2019;43:24839.

22. Ben-David K, Tuttle R, Kukar M, Rossidis G, Hochwald SN. Minimally invasive esophagectomy utilizing a stapled side-to-side anasto- mosis is safe in the western patient population. Ann Surg Oncol 2016;23:3056-62.

23. Deng XF, Liu QX, Zhou D, Min JX, Dai JG. Hand-sewn vs linearly stapled esophagogastric anastomosis for esophageal cancer: a meta-analysis. World J Gastroenterol 2015;21:4757-64.

24. Fain SN, Patin CS, Morgenstern L. Use of a mechanical suturing apparatus in low colorectal anastomosis. Arch Surg 1975;110:1079-82.

25. Van Leersum NJ, Snijders HS, Henneman D, et al. The Dutch surgical colorectal audit. Eur J Surg Oncol 2013;39:1063-70.

26. Kennedy RH, Francis EA, Wharton R, et al. Multicenter randomized controlled trial of conventional versus laparoscopic surgery for colorectal cancer within an enhanced recovery programme: EnROL. J Clin Oncol 2014;32:1804-11.

27. Vlug MS, Wind J, Hollmann MW, et al. Laparoscopy in combination with fast track multimodal management is the best perioperative strategy in patients undergoing colonic surgery: a randomized clinical trial (LAFA-study). Ann Surg 2011;254:868-75.

28. Van Oostendorp S, Elfrink A, Borstlap W, et al. Intracorporeal versus extracorporeal anastomosis in right hemicolectomy: a systematic review and meta-analysis. Surg Endosc 2017;31:64-77.

29. Kim K, Park JS, Seo H. Early outcomes of video-assisted thoracic surgery (VATS) Ivor Lewis operation for esophageal squamous cell carcinoma: the extracorporeal anastomosis technique. Surg Laparosc Endosc Percutan Tech 2013;23:303-8. 